\title{
Regulation of mutual inhibitory activities between AMPK and Akt with quercetin in MCF-7 breast cancer cells
}

\author{
YUN-KYOUNG LEE and OCK JIN PARK \\ Department of Food and Nutrition, Hannam University Daedeok Valley Campus, \\ 461-6 Jeonmin-dong, Yuseong-gu, Daejeon 305-811, Republic of Korea
}

Received June 18, 2010; Accepted August 31, 2010

DOI: $10.3892 /$ or_00001010

\begin{abstract}
In lieu of elucidating bidirectional connecting mechanism between AMP-activated protein kinase (AMPK) and survival signal Akt we applied MCF-7 breast cancer cells to determine whether AMPK modulation alters Akt signals and vice versa. Suppression of Akt activities with a synthetic Akt inhibitor alleviated AMPK activities suggesting that Akt is capable of inhibiting AMPK. Also the activation of AMPK with quercetin strongly abrogated Akt activities. Treating cancer cells with AMPK siRNA or Compound $\mathrm{C}$ resulted in marked increment of Akt dephosphorylation indicating that AMPK has antagonistic activities towards Akt. However, quercetin exerted Akt inhibitory activities in the absence of AMPK activation. Quercetin induced partial co-localization of phospho-Akt and phospho-AMPK in the nucleus even though their interaction seems to be indirect since the immunoprecipitation data indicate there was no direct binding between total Akt and AMPK. These results suggest there is a mutual suppressive interaction between AMPK and Akt. The investigation of mutual suppression between Akt and AMPK by chemo-preventive agents such as quercetin may provide a mechanistic rational for controlling breast tumor cell growth.
\end{abstract}

\section{Introduction}

Akt, a serine/threonine protein kinase, is recognized as a significant regulator of multiple cellular processes, including cell growth, survival, apoptosis and cell cycle progression (1-3). Akt is activated by phosphorylation at $\mathrm{Ser}^{473}$ or $\mathrm{Thr}^{308}$ often as a downstream sequence of phosphatidyl inositol-3 kinase (PI3K) in response to several growth factors, such as insulin-like growth factor (IGF)-1 or epidermal growth factor (EGF) (4). The aberrantly activated Akt is frequently found in many human cancers including breast cancer $(5,6)$. A

Correspondence to: Professor Ock Jin Park, Department of Food and Nutrition, Hannam University Daedeok Valley Campus, 461-6 Jeonmin-dong, Yuseong-gu, Daejeon 305-811, Republic of Korea E-mail: ojpark@hnu.kr

Key words: quercetin, AMP-activated protein kinase, Akt, mutual inhibition, MCF-7 breast cancer cells recent report suggests that Akt is a reliable biomarker for predicting the chemo-preventive effect of anti-cancer agents in breast cancer patients (7). Inhibition of apoptosis and stimulation of cell proliferation and metastasis have been shown to be carried out by the phosphorylation of Akt downstream targets including tuberous sclerosis complex 2 (TSC2)/mammalian target of rapamycin (mTOR), nuclear factor $\kappa \mathrm{B}(\mathrm{NF}-\kappa \mathrm{B}), \mathrm{Bad}$, and glycogen synthase kinase- $3 ß$ (GSK-3ß) $(8,9)$. This may lead to continuous survival of cancer cells. Therefore, regulating Akt and Akt-regulated downstream pathways has emerged as a promising strategy for controlling cancer with chemo-preventive agents.

Observations that AMPK activators are capable of dephosphorylating Akt evoked the importance of the regulation of AMPK/Akt pathway with chemo-preventive agents in cancer control (10-12). Furthermore, the Akt/TSC2/mTOR signaling pathway via the activation of AMPK has been proposed as a promising approach for tumor suppression. Several researchers have highlighted the controversial regulation between Akt and AMPK in several cancer cell models. In MDA-MB-231 cells, adiponectin-activated AMPK inhibits Akt through the activation of protein phosphatase 2A (PP2A), which directly dephosphorylates Akt (11). In other cell models of MCF-7 it was shown that the activated AMPK with anti-cancer agent blocked phosphorylation of Akt (12). On the other hand, it has been shown that Akt negatively regulates AMPK by decreasing the AMP/ATP ratio (13). It has not been resolved whether direct interaction exists between Akt and AMPK, and further mutual suppression between these two proteins in a cancer cell system challenged with anti-cancer agents. In this study, we investigated the bidirectional regulatory mechanism between Akt and AMPK in quercetin-treated MCF-7 breast cancer cells. We found that Akt and AMPK do not directly bind to each other although they partially colocalized in the nucleus. We found that the activation of AMPK resulted in the suppression of Akt, and Akt was capable of inhibiting AMPK. These observations suggest that their interaction may occur with connecting signal(s) in both of positive suppression of AMPK on Akt and the negative regulation of Akt on AMPK.

\section{Materials and methods}

Cell culture and reagents. MCF-7 cells were obtained from the American Type Culture Collection (Manassas, VA) and grown in RPMI-1640 medium containing 10\% fetal bovine 
(A)

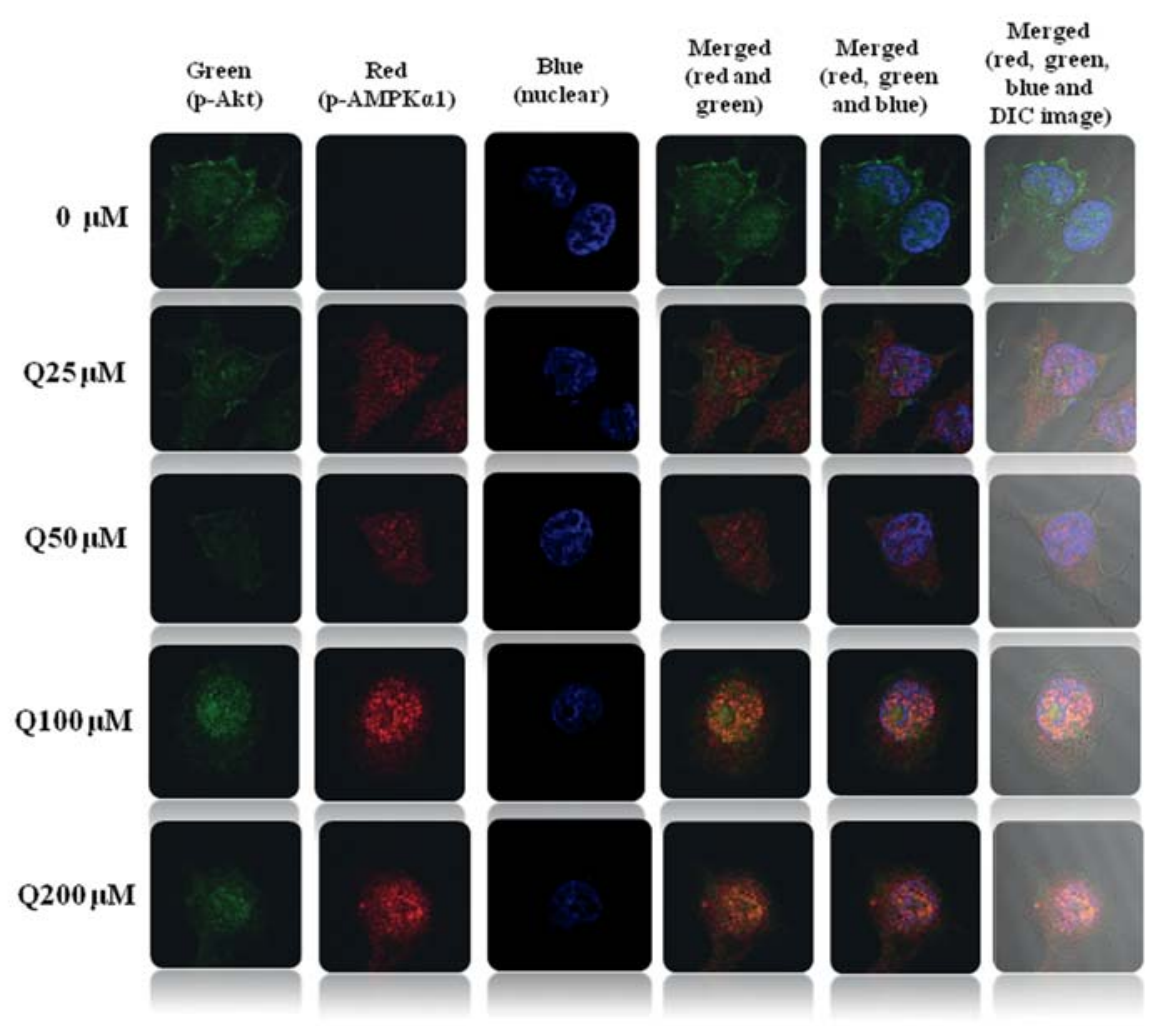

(B)

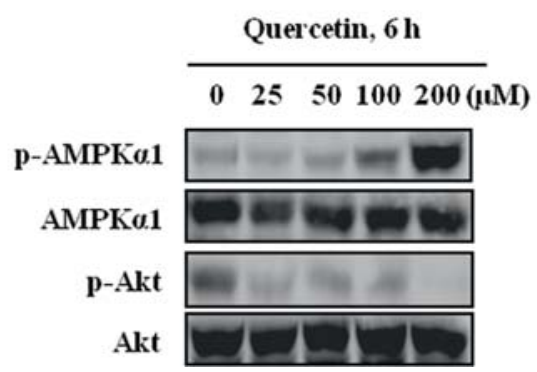

Figure 1. Quercetin decreases Akt activity and increases AMPK $\alpha 1$ activity in MCF-7 breast cancer cells. (A) The cells were treated with quercetin (0-200 $\mu \mathrm{M})$ for 6 h. Cells were fixed, permeabilized, and double stained with p-Akt (green) and anti-mouse Alexa 488 secondary antibody or with p-AMPKa1 (red) and antirabbit Alexa 546 secondary antibody. Cell nuclei were stained with Hoechst 33342 dye (blue) and observed by confocal microscope. (B) The cells were treated with quercetin $(0-200 \mu \mathrm{M})$ for $6 \mathrm{~h}$, and total proteins were subjected to Western blot analysis using p-AMPK $\alpha 1^{\text {Thr172 }}$, AMPK $\alpha 1$, p-Akt ${ }^{\text {Ser473 }}$ and Akt antibodies.

serum at $37^{\circ} \mathrm{C}$ in a $5 \% \mathrm{CO}_{2}$ atmosphere. Quercetin was purchased from Sigma-Aldrich (St. Louis, MO, USA), and Compound C was obtained from Calbiochem (San Diego, CA, USA). LY294002 was purchased from Tocris (Bristol, UK). Specific antibodies that recognize AMPK $\alpha 1$, Akt, B-actin, and the phosphorylated forms of AMPK $\alpha 1^{\text {Thr172 }}, \mathrm{ACC}^{\mathrm{Ser} 79}$ and $\mathrm{Akt}^{\mathrm{ser} 473}$ were obtained from Cell Signaling Technology (Danvers, MA, USA).

Immunofluorescence staining. Cells were seeded on a 12well plate with cover glasses. After treatment at the indicated time and dose, the cells were fixed in $3.7 \%$ formaldehyde for $20 \mathrm{~min}$ at room temperature (RT) and permeabilized in $0.2 \%$ Triton X-100 for $20 \mathrm{~min}$ at RT. Cells were blocked in $1 \%$ bovine serum albumin for $1 \mathrm{~h}$ and incubated overnight with primary antibodies directed against phospho-AMPK $\alpha 1^{\text {Thr172 }}$ and phospho-Akt ${ }^{\mathrm{ser} 473}$. After washing, the cells were incubated with Alexa 546-conjugated anti-rabbit IgG (Molecular Probes,
OR, USA) and Alexa 488-conjugated anti-mouse IgG (Molecular Probes) for $1 \mathrm{~h}$ at RT. Cell nuclei were stained with $10 \mu \mathrm{M}$ of Hoechst 33342 for 10 min and observed under a confocal microscope (Carl Zeiss, Thornwood, NY, USA).

Western blot analysis. MCF-7 breast cancer cells were seeded into 6-well plates and treated with test compounds. Total proteins were extracted using RIPA lysis buffer [50 mM Tris- $\mathrm{HCl}$ (pH 8.0), 1\% NP-40, 0.5\% sodium deoxycholate, $150 \mathrm{mM} \mathrm{NaCl}, 1 \mathrm{mM}$ PMSF] and subjected to Western blot analysis with specific antibodies. The proteins were visualized by enhanced chemiluminescence (Intron, Kyunggi, Korea) and detected using an LAS4000 chemiluminescence detection system (Fuji, Tokyo, Japan).

Transient transfection with small interfering RNA. Small interfering RNAs (siRNAs) were synthesized by Dharmacon Research (Dharmacon, Chicago, IL, USA). For transient 
transfection, the cells were seeded on a 6 -well plate $\left(2.5 \times 10^{5}\right.$ cells $/ \mathrm{ml}$ ) in antibiotic-free medium. Following an overnight incubation, the targeting siRNAs were transfected using DharmaFECT4 transfection reagent (Dharmacon) according to the manufacturer's instructions. After 72-h incubation, the cells were analyzed by Western blot analysis.

Co-immunoprecipitation assay. Cells were seeded on 60-mm culture dishes and treated with quercetin. Cells were scraped into lysis buffer (1 M Tris-HCl, 0.5 M EDTA, $1 \mathrm{M} \mathrm{NaCl}$, $1 \mathrm{M}$ DTT, $10 \% \mathrm{NP}-40,10 \mathrm{mg} / \mathrm{ml}$ BSA, 0.1 M PMSF), and the lysates were precleared with protein G-beads (Zymed, San Francisco, CA, USA) for $4 \mathrm{~h}$ at $4^{\circ} \mathrm{C}$. Proteins were then precipitated with protein G-bound primary antibody for $12 \mathrm{~h}$ at $4^{\circ} \mathrm{C}$. After washing, protein interaction was observed by Western blot analysis.

\section{Results}

Dephosphorylation of Akt and activation of AMPKal by quercetin, and partial co-localization of Akt and AMPKal in the nucleus. To examine the effects of quercetin on Akt and AMPK $\alpha 1$ activities, cells were treated with different concentrations of quercetin for $6 \mathrm{~h}$, and the levels of phospho-Akt and AMPK $\alpha 1$ were examined by immunofluorescence staining. Akt was highly activated in the membrane and cytosol of MCF-7 breast cancer cells under serumcontaining conditions, but activated Akt was abolished in a dose-dependent manner following quercetin treatment. In addition, quercetin increased the levels of phospho-AMPK $\alpha 1$ in the cytosol and nucleus, and activated AMPK $\alpha 1$ appeared to translocate to the nucleus following treatment with increasing concentrations of quercetin (Fig. 1A). Interestingly, partial co-localization was observed between phospho-Akt and phospho-AMPK $\alpha 1$ in nucleus. This is indicated by the appearance of yellow spots at concentrations of quercetin above $100 \mu \mathrm{M}$. The regulatory effects of quercetin on Akt and AMPK $\alpha 1$ were also confirmed by Western blotting. Quercetin effectively inhibited Akt through dephosphorylation but activated AMPK $\alpha 1$ in a dose-dependent manner in MCF-7 breast cancer cells (Fig. 1B).

Akt and AMPKal have mutual inhibitory activities in MCF-7 breast cancer cells. To examine the regulatory mechanism between AMPK $\alpha 1$ and Akt, MCF-7 cells were treated with specific inhibitors of Akt and AMPKa1 (LY294002 and Compound $\mathrm{C}$, respectively) and quercetin. Inhibition of AMPK $\alpha 1$ by Compound $\mathrm{C}$ increased the quercetin-induced inhibition of Akt activity compared to quercetin treatment alone, but the phospho-Akt level was lower than the control group. These results indicate that quercetin-activated AMPK $\alpha 1$ exerts inhibitory effects on Akt, and suggests that quercetin could inhibit Akt activity through an AMPK $\alpha 1$-independent pathway. In addition, treatment with LY294002 and quercetin inhibited Akt activity, but activated AMPK $\alpha 1$, more effectively than quercetin alone (Fig. 2A). Moreover, inhibition of Akt by LY294002 alone caused activation of AMPK $\alpha 1$ and phosphorylation of ACC, a substrate of AMPK $\alpha 1$ (Fig. 2B). These results suggest that Akt has inhibitory effects on AMPK $\alpha 1$ in MCF-7 breast cancer cells, and the suppression
(A)

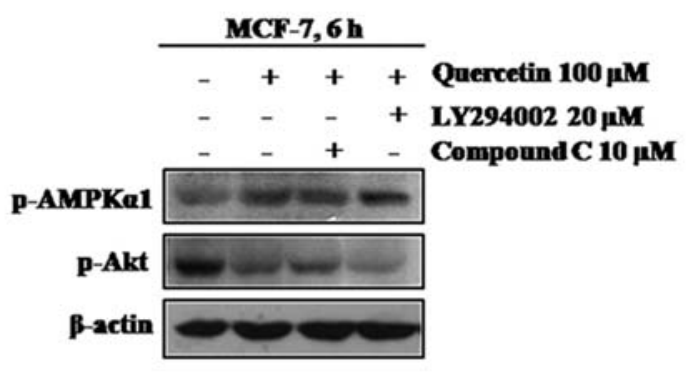

(B)

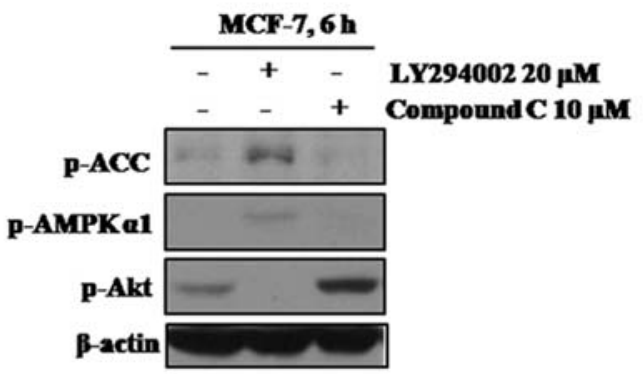

(C)

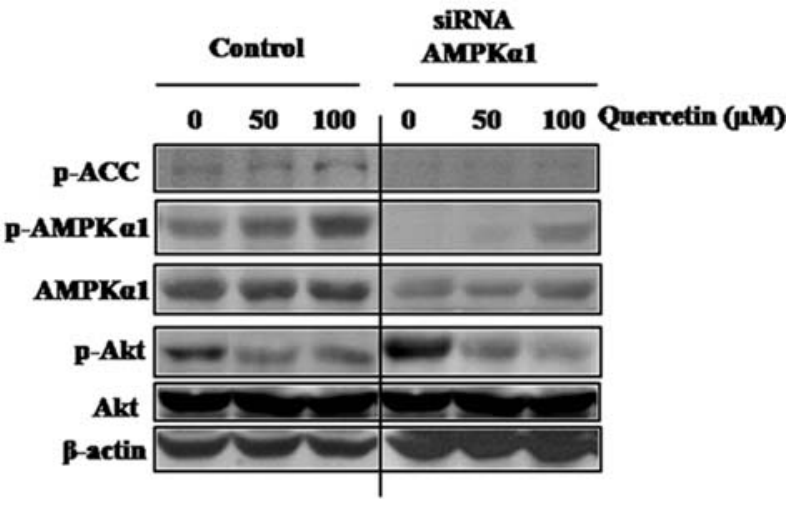

Figure 2. AMPK $\alpha 1$ and Akt exert mutual inhibitory effects in quercetintreated MCF-7 breast cancer cells. (A) The cells were pre-treated with $20 \mu \mathrm{M}$ of LY294002 or $10 \mu \mathrm{M}$ of Compound C for 30 min and then treated with $100 \mu \mathrm{M}$ of quercetin for $6 \mathrm{~h}$. Proteins were subjected Western blot analysis using the indicated antibodies. (B) The cells were treated with $20 \mu \mathrm{M}$ of LY294002 or $10 \mu \mathrm{M}$ of Compound $\mathrm{C}$ for $6 \mathrm{~h}$, and the molecular levels were examined by Western blot analysis. (C) The cells were transiently transfected with AMPK $\alpha 1$ siRNA or non-specific siRNA for $72 \mathrm{~h}$. Transfected cells or MCF-7 cells were treated with quercetin $(0-100 \mu \mathrm{M})$ for $6 \mathrm{~h}$, followed by Western blot analysis using indicated antibodies.

of Akt activity leads to an increase in AMPK $\alpha 1$ activity. To determine whether quercetin activates AMPK $\alpha 1$ through suppressing Akt, we examined the effects of quercetin on Akt following suppression of AMPK $\alpha 1$ by siRNA. Quercetin strongly inhibited Akt activity in the absence of AMPK $\alpha 1$ (Fig. 2C). Taken together, these results suggest that quercetin activates AMPK $\alpha 1$ by removing the inhibitory effects of Akt, and quercetin-activated AMPK $\alpha 1$ suppresses Akt.

Direct binding between Akt and AMPKal proteins was not observed. To investigate whether the regulatory effects that occur between AMPK $\alpha 1$ and Akt are due to their direct binding, we performed a co-immunoprecipitation assay using Akt or AMPKa1 specific antibodies. We found that Akt 
(A)

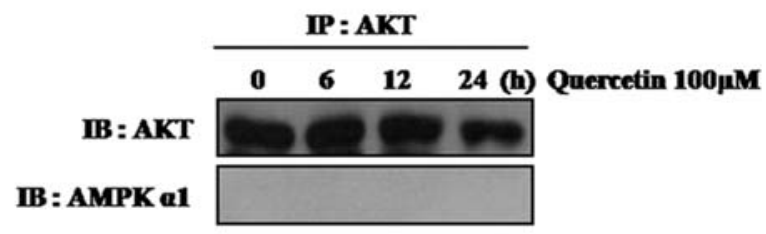

(B)

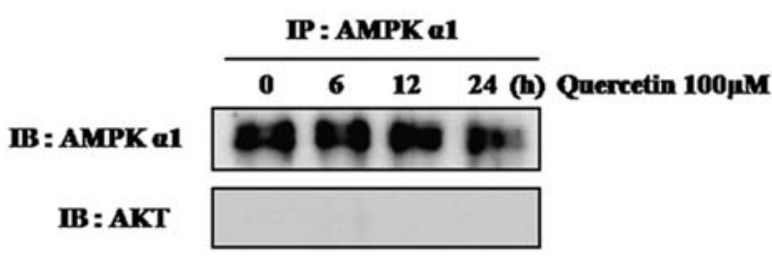

(C)

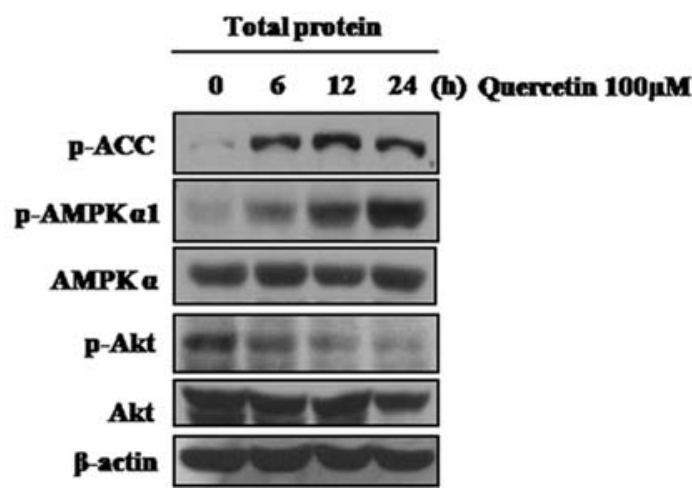

Figure 3. There is no direct binding between Akt and AMPK $\alpha 1$ in quercetintreated MCF-7 breast cancer cells. The cells were treated with $100 \mu \mathrm{M}$ of quercetin for $6,12,24 \mathrm{~h}$, and proteins were extracted. (A) Akt protein was precipitated with primary antibody and G-protein beads, and binding to AMPK $\alpha 1$ was detected by Western blot analysis using an AMPK $\alpha 1$ primary antibody. (B) AMPK $\alpha 1$ protein was precipitated with primary antibody and G-protein beads, and binding to Akt was detected by Western blot analysis using an Akt primary antibody. (C) The cells were treated with $100 \mu \mathrm{M}$ of quercetin for $6,12,24 \mathrm{~h}$, and total proteins were subjected to Western blot analysis.

and AMPK $\alpha 1$ do not bind directly (Fig. 3A and B), but that quercetin effectively inhibited Akt and activated AMPKa1 in a time-dependent manner (Fig. $3 \mathrm{C}$ ). We propose that the highly activated Akt signals observed in normal MCF-7 breast cancer cells may indicate inhibitory activity on AMPK $\alpha 1$ (Fig. 4A). In addition, quercetin could activate AMPK $\alpha 1$ by suppressing Akt activity or other Akt-independent pathways. Additionally, activated AMPKa1 is also involved in suppressing Akt activity, which may contribute to the apoptotic effects of quercetin in breast cancer (Fig. 4B).

\section{Discussion}

Quercetin, a well-investigated flavonoid, effectively inhibits cell proliferation and cell cycle progression and induces apoptosis in various cancer cells (14-17). We previously reported that quercetin inhibits cell growth and induces apoptosis in MCF-7 cells through strong activation of AMPK that suppresses the expression of the anti-apoptotic molecule
(A)

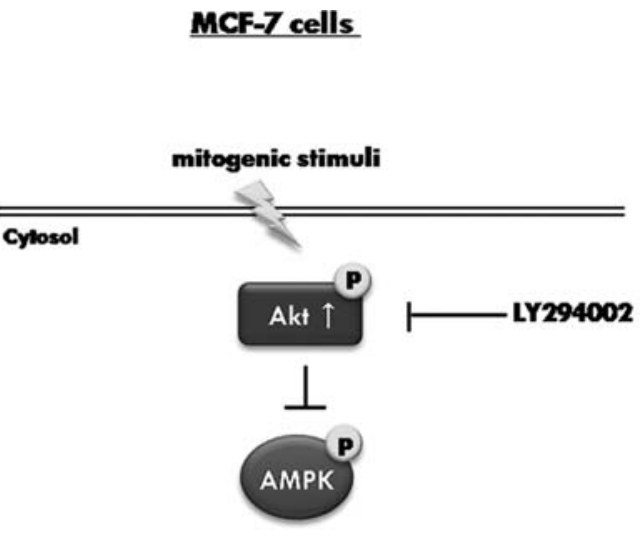

(B)

Quercetin-treated MCF-7 cells

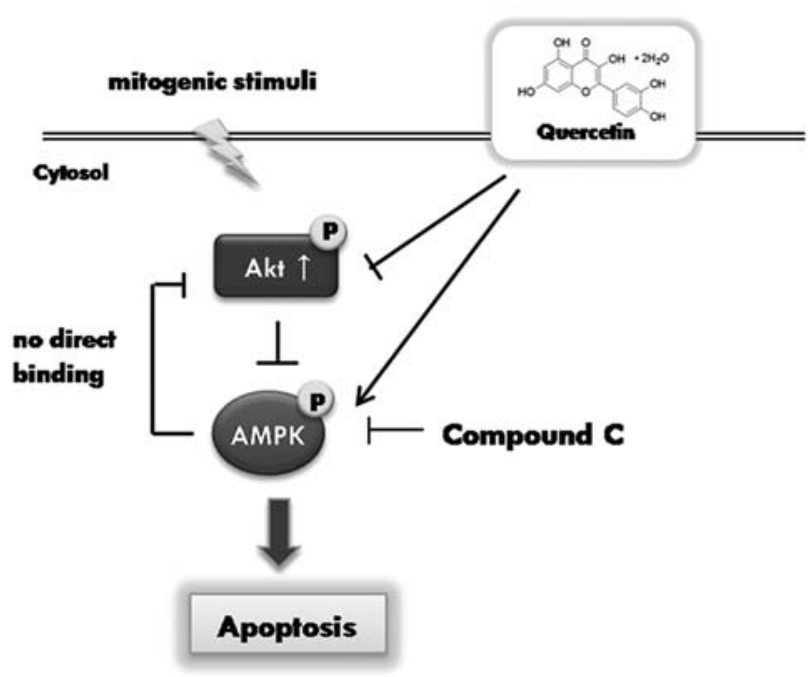

Figure 4. The regulation of Akt and AMPK $\alpha 1$ signaling by quercetin in $\mathrm{MCF}-7$ breast cancer cells.

Cox-2 (18). Quercetin also induces apoptosis in several types of cancer cells by suppressing Akt-mediated anti-apoptotic activity, cell cycle progression, and cell survival-related pathways $(15,19)$. In this study, we investigated the regulatory mechanism by which quercetin alters Akt and AMPK, two important apoptosis-regulating molecules, and the mutual regulatory mechanism between Akt and AMPK signals in quercetin-treated MCF-7 breast cancer cells, and our data indicated that quercetin was capable of activating AMPK by removing inhibitory activity of Akt on AMPK, and by direct activation on AMPK. These observations imply that Akt and AMPK have mutual inhibitory effects, and quercetin modulates both of these molecules through up- or downregulating ability in controlling cell growth or apoptosis.

Recent reports have shown that suppression of Akt by AMPK is mediated through the activation of phosphatase and tensin homologue (PTEN) or PP2A, and the involvement of IRS-1 between Akt and AMPK was suggested $(11,12,20)$. On the other hand, the inhibitory effect of Akt on AMPK 
was explained by either alteration of intracellular ATP by Akt or the regulatory activities of Akt on AMPK upstream kinases or phosphatases $(13,21)$. Our results confirmed that Akt and AMPK signals have mutual inhibitory activities in MCF-7 cell system. Treating MCF-7 cells with Akt inhibitor LY294002 or quercetin activated AMPK. The combination of quercetin and LY294002 inhibited Akt while activating AMPK more strongly than quercetin treatment alone. The activated AMPK by quercetin exerted inhibitory effects on Akt as observed by elevated phosphorylation of Akt following inhibition of AMPK by Compound C.

Because of the bidirectional relationship that occurs between Akt and AMPK following quercetin treatment, we hypothesized that quercetin may cause these two proteins to bind to each other. Immunofluorescence staining showed slight co-localization of Akt and AMPK $\alpha 1$ in the nucleus of quercetin-treated MCF-7 cells. However, co-immunoprecipitation revealed that Akt and AMPK do not bind to each other. These results suggest that there might exist another signaling molecule such as phosphatase down-regulating Akt and up-regulating AMPK activities by quercetin.

In conclusion, we demonstrated that Akt and AMPK have mutual inhibitory activities, and quercetin regulated these signals through suppressing or stimulating their actions in MCF-7 breast cancer cells. These findings imply that quercetin strongly inhibits Akt activity, possibly through activating AMPK as well as through directing its suppressive effects against Akt. These observations suggest that anticancer agents, such as quercetin, have regulatory effects on both Akt and AMPK, and this could provide an effective strategy for preventing different types of cancers, including breast cancer.

\section{Acknowledgements}

This study was supported by the Korea Science and Engineering Foundation (KOSEF) grant funded by the Korea government (MEST) (No. R01-2008-000-20131-0).

\section{References}

1. Carnero A: The PKB/AKT pathway in cancer. Curr Pharm Des 16: 34-44, 2010.

2. Bhaskar PT and Hay N: The two TORCs and Akt. Dev Cell 12: 487-502, 2007

3. Tokunaga E, Oki E, Egashira A, et al: Deregulation of the Akt pathway in human cancer. Curr Cancer Drug Targets 8: 27-36, 2008.
4. Frech M, Andjelkovic M, Ingley E, Reddy KK, Falck JR and Hemmings BA: High affinity binding of inositol phosphates and phosphoinositides to the pleckstrin homology domain of RAC/protein kinase B and their influence on kinase activity. J Biol Chem 272: 8474-8481, 1997.

5. Ghayad SE and Cohen PA: Inhibitors of the PI3K/Akt/mTOR pathway: new hope for breast cancer patients. Recent Pat Anticancer Drug Discov 5: 29-57, 2010.

6. Cicenas J: The potential role of Akt phosphorylation in human cancers. Int J Biol Markers 23: 1-9, 2008.

7. Yang SX, Costantino JP, Kim C, et al: Akt phosphorylation at Ser473 predicts benefit of paclitaxel chemotherapy in nodepositive breast cancer. J Clin Oncol 28: 2974-2981, 2010.

8. Mitsiades CS, Mitsiades N and Koutsilieris M: The Akt pathway: molecular targets for anti-cancer drug development. Curr Cancer Drug Targets 4: 235-256, 2004.

9. Wang X, Chen W and Lin Y: Sensitization of TNF-induced cytotoxicity in lung cancer cells by concurrent suppression of the NF-kappaB and Akt pathways. Biochem Biophys Res Commun 355: 807-812, 2007.

10. Eto I: Upstream molecular signaling pathways of p27(Kip1) expression: effects of 4-hydroxytamoxifen, dexamethasone, and retinoic acids. Cancer Cell Int 10: 3, 2010.

11. Kim KY, Baek A, Hwang JE, et al: Adiponectin-activated AMPK stimulates dephosphorylation of AKT through protein phosphatase 2A activation. Cancer Res 69: 4018-4026, 2009.

12. Zakikhani M, Blouin MJ, Piura E and Pollak MN: Metformin and rapamycin have distinct effects on the AKT pathway and proliferation in breast cancer cells. Breast Cancer Res Treat 123: 271-279, 2010.

13. Hahn-Windgassen A, Nogueira V, Chen CC, Skeen JE, Sonenberg $\mathrm{N}$ and Hay $\mathrm{N}$ : Akt activates the mammalian target of rapamycin by regulating cellular ATP level and AMPK activity. J Biol Chem 280: 32081-32089, 2005.

14. Kim YH and Lee YJ: TRAIL apoptosis is enhanced by quercetin through Akt dephosphorylation. J Cell Biochem 100: 998-1009, 2007.

15. Granado-Serrano AB, Angeles Martin M, Bravo L, Goya L and Ramos S: Time-course regulation of quercetin on cell survival/ proliferation pathways in human hepatoma cells. Mol Nutr Food Res 52: 457-464, 2008.

16. Zhang Q, Zhao XH and Wang ZJ: Flavones and flavonols exert cytotoxic effects on a human oesophageal adenocarcinoma cell line (OE33) by causing G2/M arrest and inducing apoptosis. Food Chem Toxicol 46: 2042-2053, 2008.

17. Park CH, Chang JY, Hahm ER, Park S, Kim HK and Yang CH: Quercetin, a potent inhibitor against beta-catenin/Tcf signaling in SW480 colon cancer cells. Biochem Biophys Res Commun 328: 227-234, 2005.

18. Lee YK, Park SY, Kim YM, Lee WS and Park OJ: AMP kinase/cyclooxygenase-2 pathway regulates proliferation and apoptosis of cancer cells treated with quercetin. Exp Mol Med 41: 201-207, 2009

19. Waite KA, Sinden MR and Eng C: Phytoestrogen exposure elevates PTEN levels. Hum Mol Genet 14: 1457-1463, 2005.

20. Huang X, Wullschleger S, Shpiro N, et al: Important role of the LKB1-AMPK pathway in suppressing tumorigenesis in PTEN-deficient mice. Biochem J 412: 211-221, 2008.

21. Kovacic S, Soltys CL, Barr AJ, Shiojima I, Walsh K and Dyck JR: Akt activity negatively regulates phosphorylation of AMP-activated protein kinase in the heart. J Biol Chem 278: 39422-39427, 2003. 